\title{
Thalamus and Cortex: Inseparable Partners in Shaping Sleep Slow Waves?
}

\author{
Francois David ${ }^{1}$ and ${ }^{\odot}$ Joscha T. Schmiedt $^{2}$ \\ ${ }^{1}$ Neuroscience Division, School of Biosciences, Cardiff University, Cardiff CF10 3AX, United Kingdom, and ${ }^{2}$ Ernst Strüngmann Institute for Neuroscience in \\ Cooperation with Max Planck Society, 60528 Frankfurt, Germany \\ Review of Lemieux et al.
}

Sleep slow waves have gained increasing attention since the pioneering work of Steriade et al. (1993a,b) unraveled their underlying cellular mechanism, i.e., the slow $(<1 \mathrm{~Hz})$ and sometimes rhythmic alternation of the membrane potential between depolarized (up) and hyperpolarized (down) states. Since then, it has been discovered that slow waves can emerge in cortical and subcortical brain areas (Crunelli and Hughes, 2010), and it has been proposed that they have important roles in the consolidation of memory (Marshall et al., 2006).

In vitro studies (Sanchez-Vives and McCormick, 2000; Crunelli and Hughes, 2010) have identified some of the ionic and synaptic mechanisms critical for the emergence of slow oscillations at both the cortical and thalamic levels. However, how in vivo network mechanisms generate the often dynamic and complex physiology of sleep slow waves remained largely elusive. How can slow waves be either local or generalized across brain areas? Why do their frequency and regularity vary? Is there only one site of initiation, and if so where? The recent study by

\footnotetext{
Received June 2, 2014; revised July 7, 2014; accepted July 11, 2014.

F.D. was supported by Epilepsy Research UK Grant No. P1202.

Correspondence should be addressed to either of the following: Francois David, Neuroscience Division, School of Biosciences, Cardiff University, Museum Avenue, Cardiff CF10 3AX, United Kingdom, E-mail: davidfo@cardiff.ac.uk; or Joscha T. Schmiedt, ErnstStrüngmann Institute for Neuroscience in Cooperation with Max Planck Society, Deutschordenstrasse 46, 60528 Frankfurt, Germany, E-mail: joscha.schmiedt@esi-frankfurt.de.

DOl:10.1523/JNEUROSCI.2226-14.2014

Copyright $\odot$ 2014 the authors $\quad 0270-6474 / 14 / 3411517-02 \$ 15.00 / 0$
}

Lemieux et al. (2014) bridges the gap between theories attributing an exclusive origin of slow waves to the cortex (Steriade et al., 1993b; Sanchez-Vives and McCormick, 2000) and contradictory observations involving other structures, especially the thalamus (Crunelli and Hughes, 2010; David et al., 2013).

In Steriade et al.'s (1993b) early work on the cellular mechanisms of the slow oscillation (and slow waves), a crucial experiment suggested that the thalamus did not play a role in the generation or upkeep of the slow oscillation: recordings performed in cats $2 \mathrm{~d}$ after the thalamus had been lesioned still revealed robust slow oscillations in cortex. This was reinforced by later experiments in vitro in cortical slices and in vivo in cortical slab (i.e., a deafferented piece of cortex maintained in situ), which showed that some of the main dynamic features typical of slow oscillations were still present in these isolated pieces of cortex (for review, see Crunelli and Hughes, 2010). In the same species and under similar anesthesia as in Steriade et al. (1993b), but contrary to the earlier findings, Lemieux et al. (2014) now show that the deafferentation of cortex, i.e., the removal of the thalamic input, induces a drastic reduction in the occurrences of slow waves and corresponding up and down states. This reduction occurred both after chemical deafferentation [via thalamic microinjection of lidocaine (QX-314); Lemieux et al., 2014, their Figs.
1-4 and 6] and after surgical isolation of cortical tissue in a slab (their Fig. 5).

Why did Lemieux et al. (2014) obtain a different result than Steriade and colleagues (1993b) had 21 years before? In contrast to Steriade et al. (1993b), Lemieux et al. (2014) were continuously monitoring cortical slow wave activity after thalamic inactivation. This revealed that within $12 \mathrm{~h}$ after deafferentation of the cortex from the thalamus, slow wave activity started to recover and was nearly fully recovered after $30 \mathrm{~h}$. Thus, the cortical circuit that generates slow waves can quickly compensate for the loss of synaptic input. A possible explanation for Steriade et al.'s (1993b) result might therefore be that at the time of their recordings ( $2 \mathrm{~d}$ after the thalamic lesion), a homeostatic process operating in the cortex had already recovered the slow wave dynamics. Given the importance of slow waves for memory consolidation, the discovery of this homeostatic plasticity mechanism that reinstates up and down dynamics might be important for understanding the functional role of sleep.

In this respect, however, one has to ask how valid a model for sleep the experiments under anesthesia are. As the various regimes and types of anesthesia generally used for the study of slow oscillations (isoflurane, ketamine-xylazine, urethane) tinker with cellular mechanisms to knock out awareness and pain, they may induce (and interact with) brain oscillations in a way that may be different in frequency, 


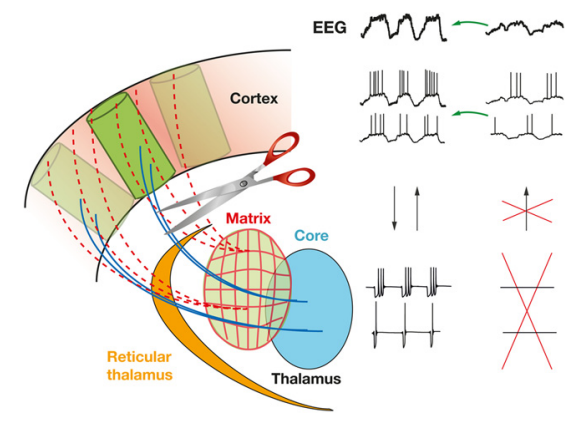

Figure 1. Slow waves in the intact brain are a product of synchronized up and down states in the thalamocortical system (traces, left column). After removal of the excitatory thalamic drive into cortex via core (diagram, solid blue lines) and matrix (diagram, dashed red lines) projections, slow wave dynamics in cortex are disrupted (traces, right column). Oscillations partially recover within $30 \mathrm{~h}$ via homeostatic processes in cortex (green arrows).

regularity, and/or synchrony from natural oscillations. However, in Lemieux et al.'s (2014) study, nonafferented areas still expressed slow waves: this result, therefore, excludes long-term effects of the anesthetic on the mechanisms generating slow waves. In addition, a different study has indeed shown a similar decrease in slow wave frequency after thalamic inactivation in naturally sleeping rats (David et al., 2013).

It is also interesting that Lemieux et al. (2014) found that not only slow waves and corresponding intracellular up and down states were affected by the cortical deafferentation (Lemieux et al., 2014, their Fig. $2 b$ ), but also activity in the frequency range above $10 \mathrm{~Hz}$ was affected. In fact, whereas the membrane potential seemed to compensate the loss of thalamic inputs with increased intracortical input (Lemieux et al., 2014, their Fig. 6) and thereby reinstated slow waves, the frequency range above $10 \mathrm{~Hz}$ did not recover. This suggests a potential role of the thalamus for the generation of higher frequency activity beyond slow wave and sleep spindle activities.

The authors also developed a computational model of the thalamocortical circuit that could reproduce their experimental observations. They proposed that upscaling of intracortical connectivity (Lemieux et al., 2014, their Figs. 9 and 10) and a change in the intrinsic/synaptic properties (their Fig. 11) of cortical neurons might be responsible for recovery from thalamic inactivation. In their model, the columnar organization of the thalamocortical circuit crucially determined how slow waves were affected (Fig. 1): the population of thalamocortical neurons with diffuse projections (i.e., matrix cells) had a much stronger impact on the amount and synchrony of cortical slow waves states than those cells that project to a single cortical column (i.e., core cells). However, in their experiment, Lemieux et al. (2014) inactivated the lateral posterior nucleus of the thalamus, which contains both diffuse and focused projections to cortex (Jones, 2001). The strong prediction of the model that diffuse projecting cells are responsible for the synchronization of slow waves across cortex, therefore, remains to be experimentally tested. If their hypothesis is correct, selective inactivation of first- versus higher-order thalamic nuclei or a cell-typespecific approach targeting core versus matrix cells (calbindin or parvalbumin immunoreactive) should reveal a differential effect on slow waves.

Finally, a question that still needs conclusive demonstration concerns the exact role played by the thalamic inputs on the slow waves during natural sleep: is it the spike timing and pattern of firing that contributes to the up state or is it the average thalamic input that helps the cortex to maintain its activity and global synchrony? Future studies should address these important questions.

\section{References}

Crunelli V, Hughes SW (2010) The slow (1 Hz) rhythm of non-REM sleep: a dialogue between three cardinal oscillators. Nat Neurosci 13:9-17. CrossRef Medline

David F, Schmiedt JT, Taylor HL, Orban G, Di Giovanni G, Uebele VN, Renger JJ, Lambert RC, Leresche N, Crunelli V (2013) Essential thalamic contribution to slow waves of natural sleep. J Neurosci 33:19599-19610. CrossRef Medline

Jones EG (2001) The thalamic matrix and thalamocortical synchrony. Trends Neurosci 24:595-601. CrossRef Medline

Lemieux M, Chen JY, Lonjers P, Bazhenov M, Timofeev I (2014) The impact of cortical deafferentation on the neocortical slow oscillation. J Neurosci 34:5689-5703. CrossRef Medline

Marshall L, Helgadóttir H, Mölle M, Born J (2006) Boosting slow oscillations during sleep potentiates memory. Nature 444:610613. CrossRef Medline

Sanchez-Vives MV, McCormick DA (2000) Cellular and network mechanisms of rhythmic recurrent activity in neocortex. Nat Neurosci 3:1027-1034. CrossRef Medline

Steriade M, Nuñez A, Amzica F (1993a) A novel slow $(<1 \mathrm{~Hz})$ oscillation of neocortical neurons in vivo: depolarizing and hyperpolarizing components. J Neurosci 13:3252-3265. Medline

Steriade M, Nuñez A, Amzica F (1993b) Intracellular analysis of relations between the slow $(<1 \mathrm{~Hz})$ neocortical oscillation and other sleep rhythms of the electroencephalogram. J Neurosci 13:3266-3283. Medline 